\title{
REPRESENTATIONS OF ABELIAN ALGEBRAIC GROUPS
}

\author{
R.P. LANGLANDS
}

There $^{1}$ is reason to believe that there is a close relation between the irreducible representations, in the sense of harmonic analysis, of the group of rational points on a reductive algebraic group over a local field and the representations of the Weil group of the local field in a certain associated complex group. There should also be a relation, although it will not be so close, between the representations of the global Weil group in the associated complex group and the representations of the adèle group that occur in the space of automorphic forms. The nature of these relations will be explained elsewhere. For now all I want to do is explain and prove the relations when the group is abelian. I should point out that this case is not typical. For example, in general there will be representations of the algebraic group not associated to representations of the Weil group.

The proofs themselves are merely exercises in class field theory. I am writing them down because it is desirable to confirm immediately the general principle, which is very striking, in a few simple cases. Moreover, it is probably impossible to attack the problem in general without having first solved it for abelian groups. If the proofs seem clumsy and too insistent on simple things remember that the author, to borrow a metaphor, has not cocycled before and has only minimum control of his vehicle.

It is well known that there is a one-to-one correspondence between isomorphism classes of algebraic tori defined over a field $F$ and split over the Galois extension $K$ of $F$ and equivalence classes of lattices on which $\mathfrak{G}(K / F)$ acts. If $T$ corresponds to $L$ then $T_{K}$, the group of $K$-rational points on $T$, may, and shall, be identified as a $\mathfrak{G}(K / F)$-module with $\operatorname{Hom}\left(L, K^{*}\right)$. If $K$ is a global field and $\mathbb{A}(K)$ is the adèle ring of $K$ the group $T_{\mathbb{A}(K)} / T_{K}$ may be identified with $\operatorname{Hom}\left(L, C_{K}\right)$ if $C_{K}$ is the idèle class group of $K$. If $K$ is a local field $C_{K}$ will be the multiplicative group of $K$.

Suppose $\widehat{L}$ is the lattice $\operatorname{Hom}(L, \mathbb{Z})$. If $\mathbb{C}^{*}$ is the multiplicative group of nonzero complex numbers and $\mathbb{C}_{u}^{*}$ the group of complex numbers of absolute

\footnotetext{
${ }^{1}$ The present paper is a reproduction, with only trivial stylistic changes, of a preprint now in circulation for 29 years. The material has, in the meantime, been treated in print by several other mathematicians. So the author is flattered that the editors of this collection still think it of some interest and is of course very pleased to be able to dedicate it to the memory of Olga Taussky-Todd.
} 
value 1 we set $\widehat{T}=\operatorname{Hom}\left(\widehat{L}, \mathbb{C}^{*}\right)$ and $\widehat{T}_{u}=\operatorname{Hom}\left(\widehat{L}, \mathbb{C}_{u}^{*}\right)$. There are natural actions of $\mathfrak{G}(K / F)$ on $\widehat{L}, \widehat{T}$, and $\widehat{T}_{u}$. The semi-direct product $\widehat{T} \rtimes \mathfrak{G}(K / F)$ is a complex Lie group with $\widehat{T}_{u} \rtimes \mathfrak{G}(K / F)$ as a real subgroup. If $F$ is a local or global field the Weil group $W_{K / F}$ is an extension

$$
0 \rightarrow C_{K} \rightarrow W_{K / F} \stackrel{\sigma}{\rightarrow} \mathfrak{G}(K / F) \rightarrow 0
$$

of $\mathfrak{G}(K / F)$ by $C_{K}$. We want to consider continuous homorphisms $\varphi$ of $W_{K / F}$ into $\widehat{T} \rtimes \mathfrak{G}(K / F)$ that make

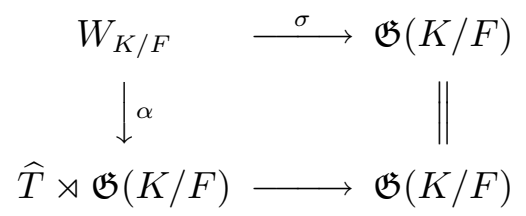

commutative. Two such homomorphisms $\varphi$ and $\varphi^{\prime}$ are said to be equivalent if there is a $t$ in $\widehat{T}$ such that $\varphi^{\prime}(w) \equiv t \varphi(w) t^{-1}$. We write $\varphi(w)$ as $f(x) \times \sigma(w)$. Then $f$ is a continuous 1 -cocycle on $W_{K / F}$ with values in $\widehat{T}$. If $\varphi^{\prime}(w)=$ $f^{\prime}(w) \times \sigma(w)$, then $\varphi$ and $\varphi^{\prime}$ are equivalent if and only if $f$ and $f^{\prime}$ are cohomologous. Thus the collection of equivalence classes may be identified with the collection $H_{c}^{1}\left(W_{K / F}, \widehat{T}\right)$ of continuous cohomology classes.

Theorem 1. If $K$ is a global or a local field there is a canonical isomorphism of $H_{c}^{1}\left(W_{K / F}, \widehat{T}\right)$ with the group of generalized characters of $\operatorname{Hom}_{\mathfrak{G}(K / F)}\left(L, C_{K}\right)$. Under this isomorphism $H_{c}^{1}\left(W_{K / F}, \widehat{T}_{u}\right)$ will correspond to the group of ordinary characters.

Suppose $F$ and $F^{\prime}$ are global or local fields, $K$ is a Galois extension of $F, K^{\prime}$ a Galois extension of $F^{\prime}$, and $\varphi$ an isomorphism of $K$ into $K^{\prime}$ that takes $F$ into $F^{\prime}$. If $F$ and $F^{\prime}$ are both global or both local we want $F^{\prime}$ to be separable over the image of $F$; if $F$ is local and $F^{\prime}$ is global we want $F^{\prime}$ to be separable over the closure of the image of $F$. Under these circumstances there is associated to $\varphi$ a homomorphism

$$
\varphi_{w}: W_{K^{\prime} / F^{\prime}} \rightarrow W_{K / F}
$$

and thus a map

$$
\varphi_{w}^{*}: H_{c}^{1}\left(W_{K / F}, \widehat{T}\right) \rightarrow H_{c}^{1}\left(W_{K^{\prime} / F^{\prime}}, \widehat{T}\right) .
$$

If $K$ is a local field $\operatorname{Hom}_{\mathfrak{G}(K / F)}\left(L, C_{K}\right)$ is just $T_{f}$, the group of $F$ rational points on $T$. However, if $K$ is a global field and $I_{K}$ is the group of idèles of $K$ the exact sequence

$$
1 \rightarrow K^{*} \rightarrow I_{K} \rightarrow C_{K} \rightarrow 0
$$


leads to the exact sequence

$$
1 \rightarrow T_{F} \rightarrow T_{\mathbb{A}(F)} \rightarrow \operatorname{Hom}_{\mathfrak{G}(K / F)}\left(L, C_{K}\right) \rightarrow H^{1}\left(\mathfrak{G}(K / F), T_{K}\right) .
$$

$T_{\mathbb{A}(F)} / T_{F}$ may be regarded as a subgroup of $\operatorname{Hom}_{\mathfrak{G}(K / F)}\left(L, C_{K}\right)$. Thus we have a canonical homomorphism of $H_{c}^{1}\left(W_{K / F}, \widehat{T}\right)$ into the group of generalized characters of $T_{\mathbb{A}(F)} / T_{F}$. The following theorem is a slight improvement of these immediate consequences of the first theorem.

Theorem 2. (a) If $F$ is a local field $H_{c}^{1}\left(W_{K / F}, \widehat{T}\right)$ is canonically isomorphic to the group of generalized characters of $T_{F}$,

(b) If $F$ is a global field there is a canonical homomorphism of $H_{c}^{1}\left(W_{K / F}, \widehat{T}\right)$ onto the group of generalized characters of $T_{\mathbb{A}(F)} / T_{F}$. The kernel is finite and consists of those classes $\alpha$ such that $\varphi_{w}^{*}(\alpha)=0$ whenever $K^{\prime}$ is the completion of $K$ with respect to some valuation, $F^{\prime}$ is the closure of $F$ in $K^{\prime}$, and $\varphi: K / F \rightarrow K^{\prime} / F^{\prime}$ is the imbedding.

Of course only the second part of this theorem will need to be proved. First we prove Theorem 1.

All $\mathfrak{G}(K / F)$-modules may be viewed as $W_{K / F^{-}}$modules and hence as $C_{K^{-}}$ modules. Of course $C_{K}$ will act trivially. The cup product gives a bilinear mapping

$$
H_{1}\left(C_{K}, L\right) \times H^{0}\left(C_{K}, L\right) \rightarrow H_{1}\left(C_{K}, \mathbb{Z}\right)
$$

that commutes with the action of $\mathfrak{G}(K / F)$ on these three groups. Since $H^{0}\left(C_{K}, L\right)$ and $H_{1}\left(C_{K}, \mathbb{Z}\right)$ are isomorphic as $\mathfrak{G}(K / F)$-modules to $L$ and $C_{K}$ respectively we have a $\mathfrak{G}(K / F)$ invariant homomorphism

$$
H_{1}\left(C_{K}, \widehat{L}\right) \rightarrow \operatorname{Hom}\left(L, C_{K}\right) .
$$

Since $L$ is the direct sum of a certain number of copies of $\mathbb{Z}$ this map is an isomorphism. The restriction map

$$
\text { Res : } H_{1}\left(W_{K / F}, \widehat{L}\right) \rightarrow H_{1}\left(C_{K}, \widehat{L}\right)
$$

has an image contained in the set of $\mathfrak{G}(K / F)$-invariant elements of $H_{1}\left(C_{K}, \widehat{L}\right)$. Using standard techniques [1], we shall show that it determines an isomorphism of $H_{1}\left(W_{K / F}, \widehat{L}\right)$ and $H_{1}\left(C_{K}, \widehat{L}\right)^{\mathfrak{G}(K / F)}$ and thus an isomorphism of $H_{1}\left(W_{K / F}, \widehat{L}\right)$ and $\operatorname{Hom}_{\mathfrak{G}(K / F)}\left(L, C_{K}\right)$. This isomorphism can be used to turn $H_{1}\left(W_{K / F}, \widehat{L}\right)$ into a topological group. To complete the proof of the first theorem we show that the pairings

$$
\begin{aligned}
H_{c}^{1}\left(W_{K / F}, \widehat{T}\right) \times H_{1}\left(W_{K / F}, \widehat{L}\right) \rightarrow H_{0}\left(W_{K / F}, \mathbb{C}^{*}\right) & =\mathbb{C}^{*} \\
H_{c}^{1}\left(W_{K / F}, \widehat{T}_{u}\right) \times H_{1}\left(W_{K / F}, \widehat{L}\right) & \rightarrow H_{0}\left(W_{K / F}, \mathbb{C}_{u}^{*}\right)=\mathbb{C}_{u}^{*}
\end{aligned}
$$


associated to the natural maps $\widehat{T} \times \widehat{L} \rightarrow \mathbb{C}^{*}$ and $\widehat{T}_{u} \times \widehat{L} \rightarrow \mathbb{C}_{u}^{*}$ determine isomorphisms of $H_{c}^{1}\left(W_{K / F}, \widehat{T}\right)$ and $H_{c}^{1}\left(W_{K / F}, \widehat{T}_{u}\right)$ with the groups of generalized and ordinary characters of $H_{1}\left(W_{K / F}, \widehat{L}\right)$.

If $x: a \rightarrow x(a)$ is a 1-cycle on $C_{K}$ with values in $\widehat{L}$ we associate to it the 1cycle on $W_{K / F}$ that is 0 outside of $C_{K}$ and equal to $x$ on $C_{K}$. If $x: w \rightarrow x(w)$ is a 1-cycle on $W_{K / F}$ we associate to it the 1-cycle $\sigma \rightarrow \sum_{\sigma=\sigma(w)} x(w)$ on $\mathfrak{G}(K / F)$. Passing to homology classes we obtain a sequence

$$
H_{1}\left(C_{K}, \widehat{L}\right) \rightarrow H_{1}\left(W_{K / F}, \widehat{L}\right) \rightarrow H_{1}(\mathfrak{G}(K / F), \widehat{L}) \rightarrow 0
$$

that we shall verify is exact. Let $\left\{w_{\sigma} \mid \sigma \in \mathfrak{G}(K / F)\right\}$ be representatives of the cosets of $C_{K}$ in $W_{K / F}$. If $x: \sigma \rightarrow x(\sigma)$ is a 1-cycle on $\mathfrak{G}(K / F)$ and $y$ is the 1-cycle on $W_{K / F}$ that is equal to $x(\sigma)$ on $w_{\sigma}$ but is zero otherwise, then $x$ is the image of $y$. Thus the sequence is exact at $H_{1}(\mathfrak{G}(K / F), \widehat{L})$. To show it is exact at $H_{1}\left(W_{K / F}, \widehat{L}\right)$ we observe first of all that if $G$ is any group and $A$ is any $G$-module then a 1-cycle that vanishes off the identity is a boundary. Indeed if $x(1)=x$ while $x(g)=0$ if $g \neq 1$, let $y(h, g)=0$ unless $h=g=1$ when $y(h, g)=x$. Then

$$
d y(g)=\sum h^{-1}(h, g)-\sum y\left(g h^{-1}, h\right)+\sum y(g, h)=y(1, g)=x(g) .
$$

Consequently the composition of the maps in and out of $H_{1}\left(W_{K / F}, \widehat{L}\right)$ is 0 . Suppose $\sigma \rightarrow \sum_{\sigma(w)=\sigma} x(w)$ is the boundary of $Y(\sigma, \tau)$. Let $z(u, v)$ be the 2-chain on $W_{K / F}$ that is 0 unless $u=w_{\sigma}$ for some $\sigma$ and $v=w_{\tau}$ for some $\tau$ when $z(u, v)=y(\sigma, \tau)$. Then

$$
d z(u)=\sum_{\sigma} w_{\sigma}^{-1} z\left(w_{\sigma}, u\right)-\sum_{\sigma} z\left(u w_{\sigma}^{-1}, w_{\sigma}\right)+\sum_{\sigma} z\left(u, w_{\sigma}\right),
$$

and if $x^{\prime}=x-d z$,

$$
\begin{aligned}
\sum_{\sigma(w)=\tau} x^{\prime}(w) & =\sum_{\sigma(w)=\tau} x(w)-\left\{\sum_{\sigma} \sigma^{-1} y(\sigma, \tau)-\sum_{\sigma} y\left(\tau \sigma^{-1}, \sigma\right)+\sum_{\sigma} y(\tau, \sigma)\right\} \\
& =0
\end{aligned}
$$

Let $z^{\prime}(u, v)=0$ unless $(u, v)=\left(w_{\sigma}, a\right)$ for some $\sigma$ and some $a$ in $C_{K}$ when $z^{\prime}(u, v)=x^{\prime}\left(w_{\sigma} a\right)$. Then

$$
\begin{aligned}
d z^{\prime}(u) & =\sum_{\sigma} w_{\sigma}^{-1} z^{\prime}\left(w_{\sigma}, u\right)-\sum_{\sigma} z^{\prime}\left(w_{\sigma}, w_{\sigma}^{-1} u\right)+\sum_{a} z^{\prime}(u, a) \\
& =\sum_{\sigma} w_{\sigma}^{-1} z^{\prime}\left(w_{\sigma}, u\right)-x^{\prime}(u) .
\end{aligned}
$$


Thus $x^{\prime}+d z^{\prime}$ has support in $C_{K}$ and the class of $x$ is in the image of $H_{1}\left(C_{K}, \widehat{L}\right)$.

Let $N$ be the map

$$
\sum_{\sigma \in \mathfrak{G}(K / F)} \sigma .
$$

It takes $H_{1}\left(C_{K}, \widehat{L}\right)$ into $H_{1}\left(C_{K}, \widehat{L}\right)^{\mathfrak{G}(K / F)}$, the group of $\mathfrak{G}(K / F)$-invariant elements. Denote its image by $N\left(H_{1}\left(C_{K}, L\right)\right)$. By definition

$$
H_{1}\left(C_{K}, \widehat{L}\right)^{\mathfrak{G}(K / F)} / N\left(H_{1}\left(C_{K}, \widehat{L}\right)\right)=\widehat{H}^{0}\left(\mathfrak{G}(K / F), H_{1}\left(C_{K}, \widehat{L}\right)\right) .
$$

The group on the right is isomorphic to

$$
\widehat{H}^{0}\left(\mathfrak{G}(K / F), \operatorname{Hom}\left(L, C_{K}\right)\right)
$$

and the sequence

$$
0 \rightarrow N\left(H_{1}\left(C_{K}, \widehat{L}\right)\right) \rightarrow H_{1}\left(C_{k}, \widehat{L}\right)^{\mathfrak{G}(K / F)} \rightarrow \widehat{H}^{0}\left(\mathfrak{G}(K / F), \operatorname{Hom}\left(L, C_{K}\right)\right) \rightarrow 0
$$

is exact.

There is an obvious isomorphism of $\widehat{L} \otimes C_{K}$ with $\operatorname{Hom}\left(L, C_{K}\right)$. It sends $\widehat{\lambda} \otimes a$ to the homomorphism $\lambda \rightarrow a^{\langle\lambda, \widehat{\lambda}\rangle}$. Since

$$
H_{1}(\mathfrak{G}(K / F), \widehat{L})=\widehat{H}^{-2}(\mathfrak{G}(K / F), \widehat{L}),
$$

the cup product gives a map

$$
H_{1}(\mathfrak{G}(K / F), \widehat{L}) \times \widehat{H}^{2}\left(\mathfrak{G}(K / F), C_{K}\right) \rightarrow \widehat{H}^{0}\left(\mathfrak{G}(K / F), \operatorname{Hom}\left(L, C_{K}\right)\right)
$$

and the cup product with the fundamental class gives a homomorphism

$$
H_{1}(\mathfrak{G}(K / F), \widehat{L}) \rightarrow \widehat{H}^{0}\left(\mathfrak{G}(K / F), \operatorname{Hom}\left(L, C_{K}\right)\right),
$$

that is well known [2] to be an isomorphism. We have to show that the following diagram (I)

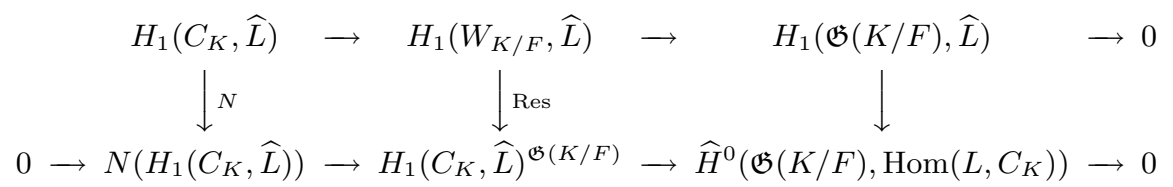

is commutative. Let $\Lambda$ be the group ring of $W_{K / F}$ over $\mathbb{Z}$ and let $I$ be the kernel of the map $\Lambda \rightarrow \mathbb{Z}$ that sends $\sum m(g) g$ to $\sum m(g)$. The exact sequence

$$
0 \rightarrow I \otimes \widehat{L} \rightarrow \Lambda \otimes \widehat{L} \rightarrow \widehat{L}=\mathbb{Z} \otimes \widehat{L} \rightarrow 0
$$


gives rise to a commutative diagram

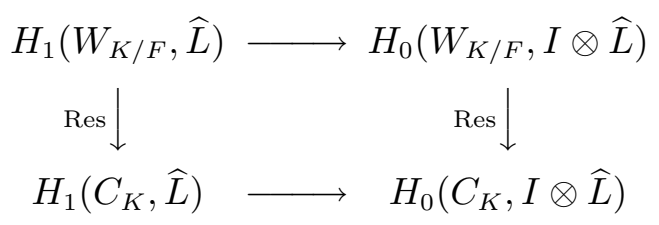

in which the horizontal arrows are isomorphisms. If $x: w \rightarrow x(w)$ is a 1-cycle on $W_{K / F}$ with values in $\widehat{L}$, the image of its homology class in $H_{0}\left(W_{K / F}, I \otimes \widehat{L}\right)$ is the class of

$$
\sum_{w}\left(w^{-1}-1\right)(1 \otimes x(w)) .
$$

Restricting to $C_{K}$ we obtain the class of

$$
\sum_{\sigma} \sum_{w}\left\{w_{\sigma} w^{-1}(1 \otimes x(w))-w_{\sigma}(1 \otimes x(w))\right\} .
$$

If $\tau$ belongs to $\mathfrak{G}(K / F)$ and $w$ belongs to $W_{K / F}$ there is a unique element $\delta\left(w_{\tau}, w\right)$ of $C_{K}$ and a unique $\sigma$ in $\mathfrak{G}(K / F)$ such that $w_{\tau} w=\delta\left(w_{\tau}, w\right) w_{\sigma}$. The sum is equal to

$$
\sum_{\tau} \sum_{w}\left(\delta^{-1}\left(w_{\tau}, w\right)-1\right) w_{\tau}(1 \otimes x(w))
$$

which equals

$$
\sum_{a \in C_{K}}\left\{\left(a^{-1}-1\right) \sum_{\delta\left(w_{\tau}, w\right)=a} w_{\tau}(1 \otimes x(w))\right\} .
$$

Since $\left(w_{\tau}-1\right) \otimes w_{\tau} x(w)$ is in $I \otimes \widehat{L}$ this is homologous to

$$
\sum_{a \in C_{K}}\left\{\left(a^{-1}-1\right) \sum_{\delta\left(w_{\tau}, w\right)=a} 1 \otimes w_{\tau} x(w)\right\} .
$$

This is the image of the class in $H_{1}\left(C_{K}, \widehat{L}\right)$ containing the 1-cycle

$$
y: a \rightarrow \sum_{\delta\left(w_{\tau}, w\right)=a} w_{\tau} x(w) .
$$

If $x$ has support in $C_{K}$,

$$
y(a)=\sum_{w_{\tau} b w_{\tau}^{-1}=a} w_{\tau} x(b)=\sum_{\tau} \tau x\left(\tau^{-1}(a)\right)
$$


and the first square at least of the diagram (I) is commutative.

In general the image of the class of $y$ in $\widehat{H}^{0}\left(\mathfrak{G}(K / F), \operatorname{Hom}\left(L, C_{K}\right)\right)$ is the class of the homomorphism

$$
\lambda \rightarrow \prod_{a} a^{\langle\lambda, y(a)\rangle}=\prod_{\tau, w} \delta\left(w_{\tau}, w\right)^{\left\langle\lambda, w_{\tau} x(w)\right\rangle} .
$$

If $w=a w_{\sigma}$ with $a$ in $C_{K}$ then $\delta\left(w_{\tau}, w\right)=w_{\tau} a w_{\tau}^{-1} \delta\left(w_{\tau}, w_{\sigma}\right)$. Thus this product equals

$$
\left\{\prod_{\sigma, \tau, a} w_{\tau} a w_{\tau}^{-1^{\left\langle\lambda, w_{\tau} x\left(a w_{\sigma}\right)\right\rangle}}\right\}\left\{\prod_{\sigma, \tau, a} \delta\left(w_{\tau}, w_{\sigma}\right)^{\left\langle\lambda, w_{\tau} x\left(a w_{\sigma}\right)\right\rangle}\right\} .
$$

The first product is a norm. Thus the homomorphism is cohomologous to

$$
\lambda \rightarrow \prod_{\sigma, \tau} \delta\left(w_{\tau}, w_{\sigma}\right)^{\langle\lambda, \tau z(\sigma)\rangle}
$$

if

$$
z(\sigma)=\sum_{a} x\left(a w_{\sigma}\right)
$$

The class of $z$ in $H_{1}(\mathfrak{G}(K / F), \widehat{L})$ is the image of the class of $x$.

To complete the verification that the diagram is commutative we have to examine the effect of taking the cup product with the fundamental class on the elements of $\widehat{H}^{-2}(\mathfrak{G}(K / F), \widehat{L})$. We proceed as at the end of Chapter XI of Serre's book [3]. We take the cup product as defined by axioms, for example, those of Serre, and by "décalage" find how it is to be expressed in terms of cycles and cocyles. Let $\Lambda$ be the group algebra of $\mathfrak{G}(K / F)$ over $\mathbb{Z}$ and let $I$ be the kernel of the map $\Lambda \rightarrow \mathbb{Z}$ that sends $\sum m(\sigma) \sigma$ to $\sum m(\sigma)$. If $B$ is a $\mathfrak{G}(K / F)$-module $\delta$ will be the isomorphism

$$
\widehat{H}^{p}(\mathfrak{G}(K / F), B) \rightarrow \widehat{H}^{p+1}(\mathfrak{G}(K / F), I \otimes B)
$$

associated to the exact sequence

$$
0 \rightarrow I \otimes B \rightarrow \Lambda \otimes B \rightarrow B \rightarrow 0 .
$$

If $A$ is another $\mathfrak{G}(K / F)$-module the following diagram (II)

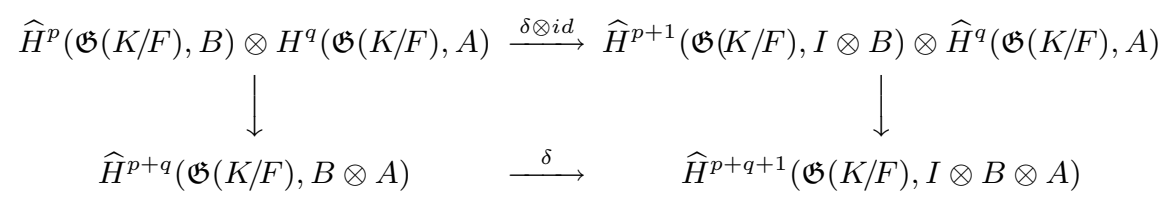


in which the vertical arrows are given by the cup product is commutative. There is also a $\mathfrak{G}(K / F)$-invariant map from $\mathbb{Z}$ to $\Lambda$. It sends $m$ to $\sum m g$. Let $J$ be the cokernel of this map. If $B$ is any $\mathfrak{G}(K / F)$-module the sequence

$$
0 \rightarrow B \rightarrow \Lambda \otimes B \rightarrow J \otimes B \rightarrow 0
$$

is exact. The associated isomorphism

$$
\widehat{H}^{p}(\mathfrak{G}(K / F), J \otimes B) \rightarrow \widehat{H}^{p+1}(\mathfrak{G}(K / F), B)
$$

will be called $d$. The following diagram (III)

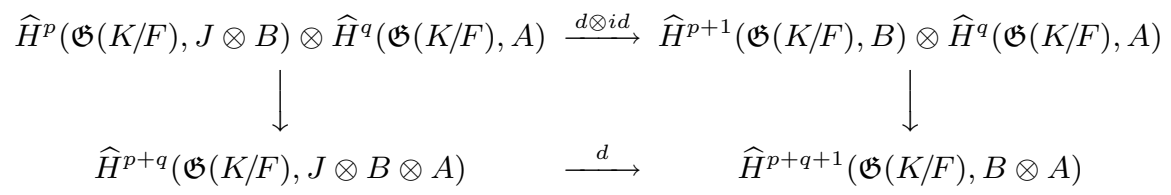

is also commutative.

If $a$ is an invariant element of $A$ the map $b \rightarrow b \otimes a$ is a $\mathfrak{G}(K / F)$ homomorphism $f_{a}$ of $B$ into $B \otimes A$. If $\bar{a}$ is the class of $a$ in $\widehat{H}^{0}(\mathfrak{G}(K / F), A)$ and $\bar{x}$ belongs to $\widehat{H}^{p}(\mathfrak{G}(K / F), B)$ the equality

$$
\bar{x} \cdot \bar{a}=f_{a}^{*}(\bar{x})
$$

is true by definition for all $B$ when $p=0$. The diagrams (I) and (II) together with the commutativity of

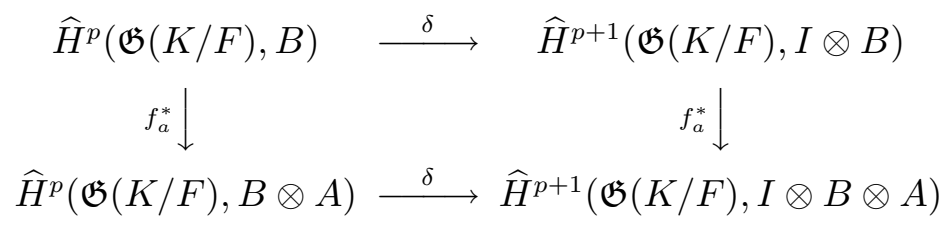

and

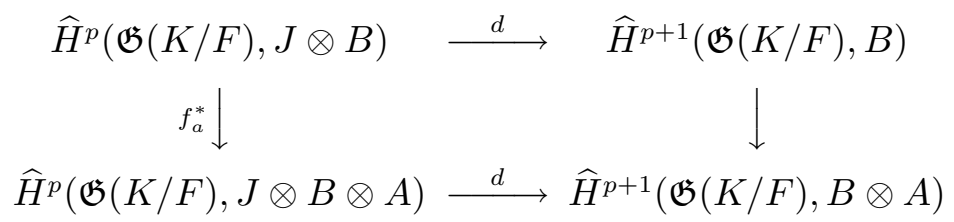

allow it to be verified, by induction up and down from 0 , for all $p$. We are going to apply the equality with the roles of $A$ and $B$ interchanged.

Suppose $a$ belongs to $A$ and $N a=0$ and $f$ is a 1-cocycle with values in $B$. We now verify that $\bar{f} \cdot \bar{a}$ is the class of $\sum_{\sigma} f(\sigma) \otimes \sigma a$ in $\widehat{H}^{0}(\mathfrak{G}(K / F), B \otimes A)$. The image of $f(\sigma)$ in $\Lambda \otimes B$ is

$$
\sum_{\tau} \tau \otimes f(\sigma)=\sum_{\tau} \sigma \tau \otimes f(\sigma)=\sum \sigma \tau \otimes f(\sigma \tau)-\sum \sigma \tau \otimes \sigma f(\tau)
$$


which, in turn, equals

$$
\sum_{\tau} \tau \otimes f(\tau)-\sigma\left(\sum_{\tau} \tau \otimes f(\tau)\right) .
$$

Thus $\bar{f}$ is the image of the class in $\widehat{H}^{0}(\mathfrak{G}(K / F), J \otimes B)$ of the image $b$ in $J \otimes B$ of $-\sum_{\tau} \tau \otimes f(\tau)$. The class of $b \otimes a$ is $\bar{b} \circ \bar{a}$. Since diagram (III) is commutative $\bar{f} \cdot \bar{a}=d(\bar{b} \cdot \bar{a})$. By the definition of $d$ on $\widehat{H}^{-1}(\mathfrak{G}(K / F), J \otimes B \otimes A)$ this is the class of the element of $B \otimes A$ corresponding to

$$
-\sum_{\sigma, \tau} \sigma \tau \otimes \sigma f(\tau) \otimes \sigma a
$$

This expression is equal to

$$
-\sum_{\sigma, \tau} \sigma \tau \otimes f(\sigma \tau) \otimes \sigma a+\sum_{\sigma, \tau} \sigma \tau \otimes f(\sigma) \otimes \sigma a .
$$

The first sum is

$$
\left(\sum_{\tau} \tau \otimes f(\tau)\right) \otimes\left(\sum_{\sigma} \sigma a\right)=0 .
$$

The second is

$$
\sum_{\tau} \tau \otimes\left(\sum_{\sigma} f(\sigma) \otimes \sigma a\right)
$$

and corresponds to $\sum_{\sigma} f(\sigma) \otimes \sigma a$ as required.

Next we verify that, if $f$ is a 1-cocycle with values in $A$ and $x$ is a 1-cycle with values in $B, \bar{x} \cdot \bar{f}$ is the class in $\widehat{H}^{-1}(\mathfrak{G}(K / F), B \otimes A)$ of $-\sum x(\sigma) \otimes f(\sigma)$. The image of this class in $\widetilde{H}^{0}(\mathfrak{G}(K / F), I \otimes B \otimes A)$ is the class of

$$
-\sum_{\tau, \sigma} \tau \otimes \tau x(\sigma) \otimes \tau f(\sigma)
$$

The image of $\bar{x}$ in $\widehat{H}^{-1}(\mathfrak{G}(K / F), I \otimes B)$ is the class of

$$
b=\sum_{\sigma}\left(\sigma^{-1}-1\right)(1 \otimes x(\sigma))
$$

Now apply the previous remark with $A$ and $B$ replaced respectively by $I \otimes B$ and $A$. Then $\bar{b} \circ \bar{f}$ is the class of

$$
-\sum_{\tau, \sigma} \tau \sigma^{-1} \otimes \tau \sigma^{-1} x(\sigma) \otimes f(\tau)+\sum_{\tau, \sigma} \tau \otimes \tau x(\sigma) \otimes f(\tau)
$$

which equals

$$
-\sum_{\tau, \sigma} \tau \otimes \tau x(\sigma) \otimes f(\tau \sigma)+\sum_{\tau, \sigma} \tau \otimes \tau x(\sigma) \otimes f(\tau)
$$


which in turn equals

$$
-\sum_{\tau, \sigma} \tau \otimes \tau x(\sigma) \otimes \tau f(\sigma)
$$

Finally we have to show that if $f$ is a 2-cocycle with values in $B$ and $x$ is 1-cycle with values in $A$ then $\bar{f} \cdot \bar{x}$ is the class of

$$
\sum_{\sigma, \tau} f(\tau, \sigma) \otimes \tau x(\sigma)
$$

The image of $f(\tau, \sigma)$ in $\Lambda \otimes B$ is

$$
\begin{aligned}
\sum_{\rho} \rho \otimes f(\tau, \sigma) & =\sum_{\rho} \tau \sigma \rho \otimes f(\tau, \sigma) \\
& =\sum \tau \sigma \rho \otimes\{\tau f(\sigma, \rho)-f(\tau \sigma, \rho)+f(\tau, \sigma \rho)\} \\
& =\tau\left(\sum \sigma \rho \otimes f(\sigma, \rho)\right)-\sum \tau \sigma \rho \otimes f(\tau \sigma, \rho)+\sum \tau \rho \otimes f(\tau, \rho) .
\end{aligned}
$$

If $g: \sigma \rightarrow \sum_{\rho} \sigma \rho \otimes f(\sigma, \rho)$ this is just $d g(\tau, \sigma)$. Thus $\bar{f}$ is the image of the class of the 1-cocycle $h$ with values in $J \otimes B$ that takes $\sigma$ to the image in $J \otimes B$ of $g(\sigma)$. The class of $-\sum h(\sigma) \otimes x(\sigma)$ in $\widehat{H}^{-1}(\mathfrak{G}(K / F), J \otimes B \otimes A)$ is $\bar{h} \cdot \bar{x}$. Applying $d$ we obtain the class of the element in $B \otimes A$ corresponding to

$$
-\sum_{\tau, \sigma, \rho} \tau \sigma \rho \otimes \tau f(\sigma, \rho) \otimes \tau x(\sigma) .
$$

This expression equals

$\sum_{\tau, \sigma, \rho} \tau \sigma \rho \otimes f(\tau \sigma, \rho) \otimes \tau x(\sigma)-\sum_{\tau, \sigma, \rho} \tau \sigma \rho \otimes f(\tau, \sigma \rho) \otimes \tau x(\sigma)+\sum_{\tau, \sigma, \rho} \tau \sigma \rho \otimes f(\tau, \sigma) \otimes \tau x(\sigma)$.

The first sum is equal to

$$
\sum_{\tau, \sigma, \rho} \tau \rho \otimes f(\tau, \rho) \otimes \tau \sigma^{-1} x(\sigma),
$$

and the second is equal to

$$
\sum_{\tau, \sigma \rho} \tau \rho \otimes f(\tau, \rho) \otimes \tau x(\sigma) .
$$

Since $\sum \sigma^{-1} x(\sigma)=\sum x(\sigma)$ their difference is 0 . The third sum is equal to

$$
\sum_{\tau, \sigma, \rho} \rho \otimes f(\tau, \sigma) \otimes \tau x(\sigma),
$$

which corresponds to

$$
\sum_{\tau, \sigma} f(\tau, \sigma) \otimes \tau x(\sigma)
$$


as required. In this case $\bar{f} \cdot \bar{x}=\bar{x} \cdot \bar{f}$ modulo the identification of $B \otimes A$ and $A \otimes B$.

Thus, if $B$ is $C_{K}$ and $f(\tau, \sigma)=\delta\left(w_{\tau}, w_{\sigma}\right)$, so that $\bar{f}$ is the fundamental class, and $A$ is $\widehat{L}$, then $\bar{x} \cdot \bar{f}$ is the class of $\sum \tau x(\sigma) \otimes \delta\left(w_{\tau}, w_{\sigma}\right)$. Applying the isomorphism of $\widehat{L} \otimes C_{K}$ with $\operatorname{Hom}\left(L, C_{K}\right)$ we obtain the homomorphism

$$
\lambda \rightarrow \prod_{\sigma, \tau} \delta\left(w_{\tau}, w_{\sigma}\right)^{\langle\lambda, \tau x(\sigma)\rangle} .
$$

The commutativity of the second square is established.

To show that

$$
\text { Res : } H_{1}\left(W_{K / F}, \widehat{L}\right) \rightarrow H_{1}\left(C_{K}, \widehat{L}\right)^{\mathfrak{G}(K / F)}
$$

is an isomorphism all we have to do is show that the kernel of the map

$$
H_{1}\left(C_{K}, \widehat{L}\right) \rightarrow H_{1}\left(W_{K / F}, \widehat{L}\right)
$$

consists precisely of the elements of norm 0 . This is equivalent to showing that the image of the adjoint map

$$
\operatorname{Hom}\left(H_{1}\left(W_{K / F}, \widehat{L}\right), \mathbb{Q} / \mathbb{Z}\right) \rightarrow \operatorname{Hom}\left(H_{1}\left(C_{K}, \widehat{L}\right), \mathbb{Q} / \mathbb{Z}\right)
$$

consists of the homomorphisms that vanish on the elements of norm 0 .

If $G$ is any group that acts on $\widehat{L}$ and $Q$ any abelian group on which $G$ acts trivially, the cup product gives a pairing

$$
H^{1}(G, \operatorname{Hom}(\widehat{L}, Q)) \times H_{1}(G, \widehat{L}) \rightarrow H_{0}(G, Q)=Q
$$

and thus a homomorphism

$$
\varphi: H^{1}(G, \operatorname{Hom}(\widehat{L}, Q)) \rightarrow \operatorname{Hom}\left(H_{1}(G, \widehat{L}), Q\right) .
$$

We need to show that $\varphi$ is an isomorphism if $Q$ is injective as an abelian group. If $f$ is a 1-cocycle and $x$ a 1 -cycle the pairing sends $\bar{f} \times \bar{x}$ to

$$
\sum_{g}\langle f(g), x(g)\rangle \text {. }
$$

Suppose $\varphi(\bar{f})=0$. Then

$$
x \rightarrow \sum\langle f(g), x(g)\rangle
$$

is a homomorphism of the group of 1-chains into $Q$ that vanishes on the cycles. Thus it defines a mapping of the boundaries in dimension 0 into $Q$. This map can be extended to a homomorphism $f^{\prime}$ of $\widehat{L}$ into $Q$. Then

$$
\sum_{g}\left\langle f(g)-d f^{\prime}(g), x(g)\right\rangle
$$


is 0 for all 1 -chains. This means $f=d f^{\prime}$ and $\bar{f}=0$. Thus $\varphi$ is injective. Conversely suppose $\psi$ is a homomorphism of $H_{1}(G, \widehat{L})$ into $Q$. Then $\psi$ determines a homomorphism of the 1-cycles into $Q$ that may be extended to a homomorphism $\psi^{\prime}$ of the group of 1-chains into $Q$. If $g$ belongs to $G$ define $f(g)$ in $\operatorname{Hom}(\widehat{L}, Q)$ by $\langle f(g), \widehat{\lambda}\rangle=\psi^{\prime}(x)$ if $x$ is the 1-chain satisfying $x(g)=\widehat{\lambda}$ and $x(h)=0$ if $h \neq g$. Then for any 1-chain $x$,

$$
\psi^{\prime}(x)=\sum_{g}\langle f(g), x(g)\rangle .
$$

Suppose $g_{1}$ and $g_{2}$ are two elements of $G$ and $x$ is the 2-chain defined by $x\left(g_{1}, g_{2}\right)=\widehat{\lambda}$, while $x(h, g)=0$ if $h \neq g_{1}$ or $g \neq g_{2}$. Then

$$
d x(g)=\sum_{h} h^{-1} x(h, g)-\sum_{h} x\left(g h^{-1}, h\right)+\sum_{h} x(g, h) .
$$

The first sum is 0 unless $g=g_{2}$ when it is $g_{1}^{-1} \widehat{\lambda}$; the second is 0 unless $g=g_{1} g_{2}$ when it is $\hat{\lambda}$; the third is 0 unless $g=g_{1}$ when it too is $\widehat{\lambda}$. Thus

$$
\begin{aligned}
0=\psi^{\prime}(d x) & =\left\langle f\left(g_{2}\right), g_{1}^{-1} \widehat{\lambda}\right\rangle-\left\langle f\left(g_{1} g_{2}\right), \widehat{\lambda}\right\rangle+\left\langle f\left(g_{1}\right), \widehat{\lambda}\right\rangle \\
& =\left\langle g_{1} f\left(g_{2}\right)-f\left(g_{1} g_{2}\right)+f\left(g_{1}\right), \widehat{\lambda}\right\rangle .
\end{aligned}
$$

Consequently $f$ is a cocycle, $\psi=\varphi(f)$, and, in conclusion, $\varphi$ is an isomorphism.

The necessary functorial properties are easily checked so that to handle the original problem we need only verify that the image of $H^{1}\left(W_{K / F}\right.$, $\operatorname{Hom}(\widehat{L}, \mathbb{Q} / \mathbb{Z}))$ in $H^{1}\left(C_{K}, \operatorname{Hom}(\widehat{L}, \mathbb{Q} / \mathbb{Z})\right)$ consists of those elements corresponding to homomorphisms vanishing on elements of norm 0 . This problem may be reformulated. $\mathfrak{G}(K / F)$ acts on $\operatorname{Hom}(\widehat{L}, \mathbb{Q} / \mathbb{Z})$, and we can form the semi-direct product $\operatorname{Hom}(\widehat{L}, \mathbb{Q} / \mathbb{Z}) \times \mathfrak{G}(K / F)$. Suppose we have a commutative diagram

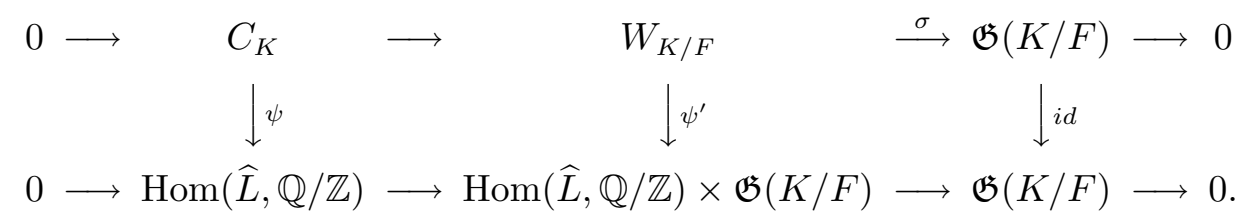

Define the 1-cochain $f$ by $\psi^{\prime}(w)=f(w) \times \sigma(w)$. Then $f$ is a cocycle. Conversely given a cocycle this equation determines a $\psi^{\prime}$ that, together with its restriction $\psi$ to $C_{K}$, will make the diagram commutative. What we have to show is that given $\psi$ we can find a $\psi^{\prime}$ making the diagram commutative if and only if the associated homomorphism $\varphi: H_{1}\left(C_{K}, \widehat{L}\right) \rightarrow \mathbb{Q} / \mathbb{Z}$ vanishes on the elements of norm 0 . It is well known $[3]$ that $\psi$ can be so extended if and only 
if it is $\mathfrak{G}(K / F)$ invariant and sends the fundamental class $H^{2}\left(\mathfrak{G}(K / F), C_{K}\right)$ to 0 . The first condition is satisfied if $\varphi$ vanishes on the elements of norm 0 because the map from $\psi$ to $\varphi$ is $\mathfrak{G}(K / F)$ invariant. We need to verify that the diagram

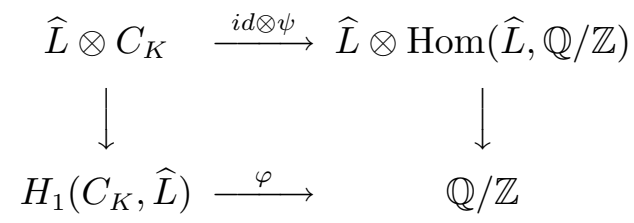

is commutative. The arrow on the left is the composition of the isomorphism of $\widehat{L} \otimes C_{K}$ with $\operatorname{Hom}\left(L, C_{K}\right)$ and $\operatorname{Hom}\left(L, C_{K}\right)$ with $H_{1}\left(C_{K}, \widehat{L}\right)$ previously introduced. It sends $\hat{\lambda} \otimes a$ to the class of the 1-cycle that is 0 except at $a$ where it is $\hat{\lambda}$. Applying $\varphi$ we obtain $\langle\psi(a), \hat{\lambda}\rangle$ which is, of course, the result obtained by moving around the diagram in the other direction. If $\psi$, and hence $\varphi$, is $\mathfrak{G}(K / F)$-invariant we are led to the commutative diagram

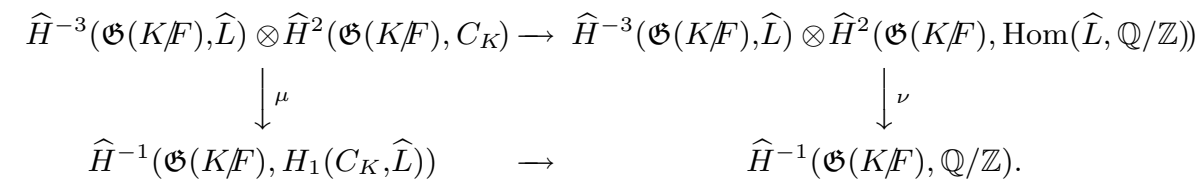

The vertical arrows are given by the cup products. If $\alpha$ is the fundamental class $\psi(\alpha)=0$ if and only if $\nu(\beta \otimes \psi(\alpha))=0$ for all $\beta$. On the other hand $\gamma \rightarrow \mu(\gamma \otimes \alpha)$ is an isomorphism of $\widehat{H}^{-3}(\mathfrak{G}(K / F), \widehat{L})$ and $\widehat{H}^{-1}\left(\mathfrak{G}(K / F), H_{1}\left(C_{K}, \widehat{L}\right)\right)$. Thus $\psi(\alpha)=0$ if and only if the lower horizontal arrow is 0 . By its definition this is so if and only if $\varphi$ vanishes on the elements of norm 0 .

We now know that $H_{1}\left(W_{K / F}, \widehat{L}\right)$ is isomorphic to $\operatorname{Hom}_{\mathfrak{G}(K / F)}\left(L, C_{K}\right)$ and that, since both $\mathbb{C}^{*}$ and $\mathbb{C}_{u}^{*}$ are injective, there are isomorphisms

$$
\begin{aligned}
\varphi: H^{1}\left(W_{K / F}, \widehat{T}\right) & \rightarrow \operatorname{Hom}\left(H_{1}\left(W_{K / F}, \widehat{L}\right), \mathbb{C}^{*}\right) \\
\varphi: H^{1}\left(W_{K / F}, \widehat{T}_{u}\right) & \rightarrow \operatorname{Hom}\left(H_{1}\left(W_{K / F}, \widehat{L}\right), \mathbb{C}_{u}^{*}\right) .
\end{aligned}
$$

We need only verify that $\varphi(\bar{f})$ is continuous if and only if $f$ is a continuous cocycle.

If $U_{K}$ consists of the elements of norm 1 in $C_{K}$ we have an exact sequence

$$
1 \rightarrow U_{K} \rightarrow C_{K} \rightarrow M_{K} \rightarrow 1
$$

$M_{K}$ is $\mathbb{Z}$ or $\mathbb{R}$ and $\mathfrak{G}(K / F)$ acts trivially on it. The sequence splits as a sequence of abelian groups and

$$
0 \rightarrow \operatorname{Hom}\left(L, U_{K}\right) \stackrel{\lambda}{\rightarrow} \operatorname{Hom}\left(L, C_{K}\right) \stackrel{\mu}{\rightarrow} \operatorname{Hom}\left(L, M_{K}\right) \rightarrow 0
$$


is exact. There is an obvious map

$$
\begin{aligned}
N\left(\operatorname{Hom}\left(L, C_{K}\right)\right) & \cap \operatorname{Hom}\left(L, U_{K}\right) / N\left(\operatorname{Hom}\left(L, U_{K}\right)\right) \\
& \rightarrow \widehat{H}^{-1}\left(\mathfrak{G}(K / F), \operatorname{Hom}\left(L, M_{K}\right)\right) / \mu \widehat{H}^{-1}\left(\mathfrak{G}(K / F), \operatorname{Hom}\left(L, C_{K}\right)\right) .
\end{aligned}
$$

If $z=N x$ belongs to $\operatorname{Hom}\left(L, U_{K}\right)$ and $y$ is the image of $x$ in $\operatorname{Hom}\left(L, M_{K}\right)$, then $N y=0$. We send $z$ to the image of $y$ in the group on the right. The image is independent of $x$ and if $x$ can be chosen in $\operatorname{Hom}\left(L, U_{K}\right)$ it is 0 . If the image is 0 we can so choose $x$ that $y=\sum_{\sigma} \sigma^{-1} v_{\sigma}-v_{\sigma}$. If $u_{\sigma}$ in $\operatorname{Hom}\left(L, C_{K}\right)$ maps to $v_{\sigma}$ then $x^{\prime}=x-\sum \sigma^{-1} u_{\sigma}-u_{\sigma}$ lies in $\operatorname{Hom}\left(L, U_{K}\right)$ and $N x^{\prime}=$ $N x$. Thus the map is an injection. Since the group on the right is finite so is the one on the left. Since $\operatorname{Hom}\left(L, U_{K}\right)$ is compact, $N\left(\operatorname{Hom}\left(L, U_{K}\right)\right)$, and hence $N\left(\operatorname{Hom}\left(L, C_{K}\right)\right) \cap \operatorname{Hom}\left(L, U_{K}\right)$, is closed in $\operatorname{Hom}\left(L, U_{K}\right)$. Since $\operatorname{Hom}_{\mathfrak{G}(K / F)}\left(L, U_{K}\right)$ is an open subgroup of $\operatorname{Hom}_{\mathfrak{G}(K / F)}\left(L, C_{K}\right)$, except when $K$ is archimedean, and

$$
N\left(\operatorname{Hom}\left(L, C_{K}\right)\right) \cap \operatorname{Hom}_{\mathfrak{G}(K / F)}\left(L, U_{K}\right)=N\left(\operatorname{Hom}\left(L, C_{K}\right)\right) \cap \operatorname{Hom}\left(L, U_{K}\right)
$$

is closed, $N\left(\operatorname{Hom}\left(L, C_{K}\right)\right)$ is closed in $\operatorname{Hom}_{\mathfrak{G}(K / F)}\left(L, C_{K}\right)$. Since it is of finite index, it is also open. In the archimedean case the sequence

$$
0 \rightarrow U_{K} \rightarrow C_{K} \rightarrow M_{K} \rightarrow 0
$$

splits as a sequence of $\mathfrak{G}(K / F)$-modules and

$$
\operatorname{Hom}_{\mathfrak{G}(K / F)}\left(L, C_{K}\right)=\operatorname{Hom}_{\mathfrak{G}(K / F)}\left(L, U_{K}\right) \times \operatorname{Hom}_{\mathfrak{G}(K / F)}\left(L, M_{K}\right) .
$$

Moreover

$$
N\left(\operatorname{Hom}\left(L, M_{K}\right)\right)=\operatorname{Hom}_{\mathfrak{G}(K / F)}\left(L, M_{K}\right),
$$

so that, in this case too, $N(\operatorname{Hom}(L, C) K)$ is closed in $\operatorname{Hom}_{\mathfrak{G}(K / F)}\left(L, C_{K}\right)$.

The upshot is that a homomorphism $\varphi$ of $\operatorname{Hom}_{\mathfrak{G}(K / F)}\left(L, C_{K}\right)$ or, what is the same, $H_{1}\left(C_{K}, \widehat{L}\right)^{\mathfrak{G}(K / F)}$ into $\mathbb{C}_{u}^{*}$ or $\mathbb{C}^{*}$ is continuous if and only if $\varphi \circ N$ is continuous. Of course an element of $H^{1}\left(W_{K / F}, \widehat{T}_{u}\right)$ or $H^{1}\left(W_{K / F}, \widehat{T}\right)$ is continuous if and only if its restriction to $C_{K}$ is. Since the diagrams

$$
\begin{aligned}
& H^{1}\left(W_{K / F}, \widehat{T}\right) \leftrightarrow \operatorname{Hom}\left(H_{1}\left(W_{K / F}, \widehat{L}\right), \mathbb{C}^{*}\right) \\
& \downarrow \\
& H^{1}\left(C_{K}, \widehat{T}\right) \leftrightarrow \operatorname{Hom}\left(H_{1}\left(C_{K}, \widehat{L}\right), \mathbb{C}^{*}\right) \\
& H^{1}\left(W_{K / F}, \widehat{T}_{u}\right) \leftrightarrow \operatorname{Hom}\left(H_{1}\left(W_{K / F}, \widehat{L}\right), \mathbb{C}_{u}^{*}\right) \\
& \begin{array}{c}
\downarrow \\
H^{1}\left(C_{K}, \widehat{T}_{u}\right) \leftrightarrow \operatorname{Hom}\left(H_{1}\left(C_{K}, \widehat{L}\right), \mathbb{C}_{u}^{*}\right)
\end{array}
\end{aligned}
$$


in which the right hand vertical arrows are adjoint to the corestriction are commutative, we need only check that $\bar{f}$ in $H^{1}\left(C_{K} \widehat{T}\right)$ is continuous if and only if the corresponding element of $\operatorname{Hom}\left(H_{1}\left(C_{K}, \widehat{L}\right), \mathbb{C}^{*}\right)$ or $\operatorname{Hom}(\widehat{L} \otimes$ $\left.C_{K}, \mathbb{C}^{*}\right)$ is continuous. Since that homomorphism sends $\hat{\lambda} \otimes a$ to $\langle\widehat{\lambda}, f(a)\rangle$ this is clear.

We have still to prove the second part of Theorem 2. The diagram

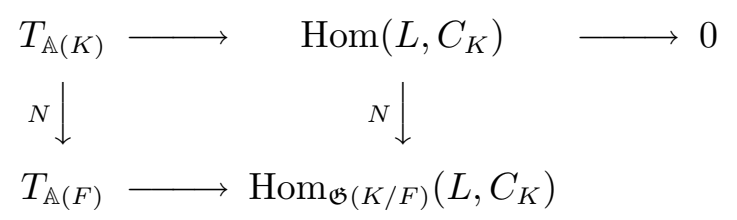

is commutative and the upper row is exact. Thus $T_{\mathbb{A}(F)} / T_{F}$ contains $N\left(\operatorname{Hom}\left(L, C_{K}\right)\right)$ and is a closed subgroup of finite index in $\operatorname{Hom}_{\mathfrak{G}(K / F)}\left(L, C_{K}\right)$.

If $K^{\prime}$ is the completion of $K$ with respect to some valuation and $F^{\prime}$ is the closure of $F$ in $K^{\prime}$ there is a mapping $T_{F^{\prime}} \rightarrow T_{\mathbb{A}(F)}$ and thus a mapping $T_{F^{\prime}} \rightarrow \operatorname{Hom}_{\mathfrak{G}(K / F)}\left(L, C_{K}\right)$. A generalized character of $\operatorname{Hom}_{\mathfrak{G}(K / F)}\left(L, C_{K}\right)$ is trivial on $T_{\mathbb{A}(F)} / T_{F}$ if and only if it is trivial on the image of $T_{F^{\prime}}$, for all choices of $K^{\prime}$.

$$
T_{F^{\prime}}=\operatorname{Hom}_{\mathfrak{G}\left(K^{\prime} / F^{\prime}\right)}\left(L, C_{K^{\prime}}\right)
$$

and, if $E=K \cap F^{\prime}$, the natural map $C_{K^{\prime}} \rightarrow C_{K}$ gives a map

$$
\operatorname{Hom}_{\mathfrak{G}\left(K^{\prime} / F^{\prime}\right)}\left(L, C_{K^{\prime}}\right) \rightarrow \operatorname{Hom}_{\mathfrak{G}(K / E)}\left(L, C_{K}\right) .
$$

If $\mathfrak{G}(K / F)$ is the disjoint union $\bigcup_{i=1}^{r} \sigma_{i} \mathfrak{G}(K / E)$ we compose this with the map $\sum_{i=1}^{r} \sigma_{1}$ of $\operatorname{Hom}_{\mathfrak{G}(K / E)}\left(L, C_{K}\right)$ into $\operatorname{Hom}_{\mathfrak{G}(K / F)}\left(L, C_{K}\right)$ to obtain the map of $T_{F^{\prime}}$ into $\operatorname{Hom}_{\mathfrak{G}(K / F)}\left(L, C_{K}\right)$.

On the other hand if $\varphi$ is the imbedding $K / F \rightarrow K^{\prime} / F^{\prime}$, the map $\varphi_{w}$ : $W_{K^{\prime} / F^{\prime}} \rightarrow W_{K / F}$ determines maps

$$
\begin{aligned}
& H_{c}^{1}\left(W_{K / F}, \widehat{T}\right) \longrightarrow H_{c}^{1}\left(W_{K^{\prime} / F^{\prime}}, \widehat{T}\right) \\
& H_{1}\left(W_{K^{\prime} / F^{\prime}}, \widehat{L}\right) \longrightarrow H_{1}\left(W_{K / F}, \widehat{L}\right)
\end{aligned}
$$

that are adjoint to each other. All we need do is verify that

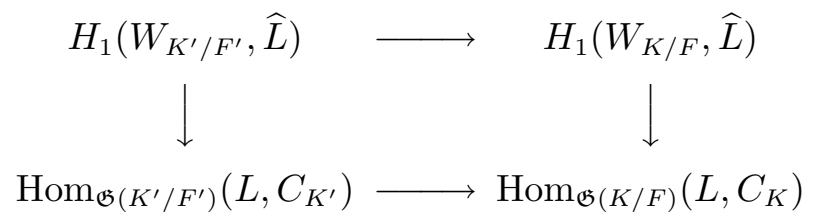

is commutative. 
Let $x^{\prime}$ be a 1-cycle on $W_{K^{\prime} / F^{\prime}}$ with values in $\widehat{L}$ and let $x$ be the 1-cycle on $W_{K / F}$ that sends $w$ to $\sum_{w^{\prime} \rightarrow w} x^{\prime}\left(w^{\prime}\right)$. Restriction to $C_{K^{\prime}}$ sends the class of $x^{\prime}$ to the class of $y^{\prime}$ if

$$
y^{\prime}\left(a^{\prime}\right)=\sum_{\delta\left(w_{\tau}, w^{\prime}\right)=a^{\prime}} w_{\tau^{\prime}} x^{\prime}\left(w^{\prime}\right) .
$$

Restriction to $C_{K}$ sends the class of $x$ to the class of $y$ if

$$
y(a)=\sum_{\delta\left(w_{\tau}, w\right)=a} w_{\tau} x(w) .
$$

The class of $y^{\prime}$ corresponds to the homomorphism

$$
\lambda \rightarrow \prod_{\tau^{\prime}} \prod_{w^{\prime}} \delta\left(w_{\tau^{\prime}}, w^{\prime}\right)^{\left\langle\lambda, w_{\tau^{\prime}} x^{\prime}\left(w^{\prime}\right)\right\rangle}
$$

and that of $y$ to the homomorphism

$$
\lambda \rightarrow \prod_{\tau} \prod_{w} \delta\left(w_{\tau}, w\right)^{\left\langle\lambda, w_{\tau} x(w)\right\rangle} .
$$

The inner product need only be taken over $W_{K / E}$.

If $\sigma=\sigma_{i} \tau$ with $\tau$ in $\mathfrak{G}(K / E)$ we take $w_{\sigma}=w_{\sigma_{i}} w_{\tau}$. Then if $w$ belongs to $W_{K / E}$

$$
w_{\sigma_{i}} w_{\tau} w=w_{\sigma_{i}} \delta\left(w_{\tau}, w\right) w_{\sigma_{i}}^{-1} w_{\sigma_{i}} w_{\rho}
$$

for some $\rho$ in $\mathfrak{G}(K / E)$. Thus the homomorphism corresponding to $y$ sends $\lambda$ to

$$
\sum_{i=1}^{r} \sigma_{i}\left(\varphi\left(\sigma_{i}^{-1} \lambda\right)\right)
$$

if $\varphi$ is the homomorphism

$$
\lambda \rightarrow \prod_{\tau \in \mathfrak{G}(K / E)} \prod_{w \in W_{K / E}} \delta\left(w_{\tau}, w\right)^{\left\langle\lambda, w_{\tau} x(w)\right\rangle}
$$

of $L$ into $C_{K}$. The homomorphism $\varphi_{w}$ is an injection and we may use it to identify $W_{K^{\prime} / F^{\prime}}$ with a subgroup of $W_{K / E}$. We may also identify $\mathfrak{G}(K / E)$ and $\mathfrak{G}\left(K^{\prime} / F^{\prime}\right)$. If $\tau^{\prime} \leftrightarrow \tau$ we take $w_{\tau^{\prime}} \leftrightarrow w_{\tau}$. If $w^{\prime} \leftrightarrow w$

$$
\delta\left(w_{\tau^{\prime}}, w^{\prime}\right) \leftrightarrow \delta\left(w_{\tau}, w\right)
$$

and the above product may be written

$$
\prod_{\tau^{\prime}} \prod_{w^{\prime}} \delta\left(w_{\tau^{\prime}}, w^{\prime}\right)^{\left\langle\lambda, w_{\tau^{\prime}} x^{\prime}\left(w^{\prime}\right)\right\rangle}
$$


so that $\varphi$ is the homomorphism corresponding to $y^{\prime}$. The diagram is commutative and Theorem 2 is proved.

It is convenient to add now one or two observations that will be used elsewhere to compare two kinds of $L$-series. Suppose $F$ is a non-archimedean local field and $K / F$ is unramified. I claim that

$$
N: \operatorname{Hom}\left(L, U_{K}\right) \rightarrow \operatorname{Hom}_{\mathfrak{G}(K / F)}\left(L, U_{K}\right)
$$

is surjective. To see this let $U_{k}^{n}=\left\{x \in U_{K} \mid x \equiv 1\left(\bmod P_{K}^{n}\right)\right\}$ if $n \geq 1$. These subgroups are $\mathfrak{G}(K / F)$-invariant and we need only verify that

$$
\begin{aligned}
N: \operatorname{Hom}\left(L, U_{K} / U_{K}^{1}\right) & \rightarrow \operatorname{Hom}_{\mathfrak{G}(K / F)}\left(L, U_{K} / U_{K}^{1}\right) \\
N: \operatorname{Hom}\left(L, U_{K}^{n} / U_{K}^{n+1}\right) & \rightarrow \operatorname{Hom}_{\mathfrak{G}(K / F)}\left(L, U_{K}^{n} / U_{K}^{n+1}\right)
\end{aligned}
$$

are surjective. Let $\kappa=O_{K} / P_{K}$ be the residue field of $O_{K}$. The group $U_{K}^{n} / U_{K}^{n+1}$ is isomorphic as a $\mathfrak{G}(K / F)$-module to $\kappa$; so we consider

$$
N: \operatorname{Hom}(L, \kappa) \rightarrow \operatorname{Hom}_{\mathfrak{G}(K / F)}(L, \kappa) .
$$

If $k=O_{F} / P_{F}$ and $\Lambda$ is the group ring of $\mathfrak{G}(K / F)$ then $\kappa$ is isomorphic as a $\mathfrak{G}(K / F)$-module to $\Lambda \otimes k$, and $\operatorname{Hom}(L, \Lambda \otimes k)$ is isomorphic to $\Lambda \otimes \operatorname{Hom}(L, k)$, so that

$$
\widehat{H}^{0}(\mathfrak{G}(K / F), \Lambda \otimes \operatorname{Hom}(L, k))=0
$$

and $N$ is surjective. The group $U_{K} / U_{K}^{1}$ is isomorphic as a $\mathfrak{G}(K / F)$ module to $\kappa^{*}$ the multiplicative group of $\kappa$; so we consider

$$
N: \operatorname{Hom}\left(L, \kappa^{*}\right) \rightarrow \operatorname{Hom}_{\mathfrak{G}(K / F)}\left(L, \kappa^{*}\right) .
$$

To show this is surjective is the same as showing that $\widehat{H}^{0}(\mathfrak{G}(K / F)$, $\left.\operatorname{Hom}\left(L, \kappa^{*}\right)\right)$ is 0 . Since $\mathfrak{G}(K / F)$ is cyclic and $\operatorname{Hom}\left(L, \kappa^{*}\right)$ is finite all the groups $\widehat{H}^{p}\left(\mathfrak{G}(K / F), \operatorname{Hom}\left(L, \kappa^{*}\right)\right)$ have the same order. We show that

$$
\widehat{H}^{1}\left(\mathfrak{G}(K / F), \operatorname{Hom}\left(L, \kappa^{*}\right)\right)=H^{1}\left(\mathfrak{G}(K / F), \operatorname{Hom}\left(L, \kappa^{*}\right)\right)
$$

is 0 . If $\bar{\kappa}$ is the algebraic closure of $\kappa$ and $\mathfrak{G}$ is the subgroup of $\mathfrak{G}(\bar{\kappa} / \kappa)$ generated by the Frobenius substitution, the sequence

$$
0 \rightarrow H^{1}\left(\mathfrak{G}(K / F), \operatorname{Hom}\left(L, \kappa^{*}\right)\right) \rightarrow H^{1}\left(\mathfrak{G}, \operatorname{Hom}\left(L, \bar{\kappa}^{*}\right)\right)
$$

is exact. We show that the group on the right is 0 . If $\sigma_{0}$ is the Frobenius substitution any 1 -cycle $f$ on $\mathfrak{G}$ is determined by $f\left(\sigma_{0}\right)$. To prove that $f$ is a 
boundary we have to show that $f\left(\sigma_{0}\right)=\sigma_{0} \varphi-\varphi$ for some $\varphi$ in $\operatorname{Hom}\left(L, \bar{\kappa}^{*}\right)$. Let $\lambda_{1}, \cdots, \lambda_{n}$ be a basis for $L$ and let

$$
\sigma_{0}^{-1}=\sum_{j} a_{j i} \lambda_{j}
$$

The matrix $\left(a_{i j}\right)$ is integral and all its eigenvalues are algebraic integers. Thus, if $q$ is the number of elements in $\kappa,\left(q a_{i j}-\delta_{i j}\right)$, where $\delta_{i j}$ is Kronecker's delta, is non-singular. We may write it as a product $\left(b_{i j}\right)\left(m_{i} \delta_{i j}\right)\left(c_{i j}\right)$ where $\left(b_{i j}\right)$ and $\left(c_{i j}\right)$ are integral with integral inverses $\left(b_{i j}^{\prime}\right)$ and $\left(c_{i j}^{\prime}\right)$, and $m_{1}, \cdots, m_{n}$ are integers. Let $f\left(\sigma_{0}\right)=\psi$ and let $\psi\left(\lambda_{i}\right)=\alpha_{i}$. If $\varphi\left(\lambda_{i}\right)=\beta_{i}$,

$$
\sigma_{0} \varphi\left(\lambda_{i}\right)=\sigma_{0}\left(\varphi\left(\sigma^{-1} \lambda_{i}\right)\right)=\prod_{j=1}^{n} \beta_{j}^{q a_{j i}}
$$

and the equation $\psi=\sigma_{0} \varphi-\varphi$ is equivalent to the $n$ equations:

$$
\alpha_{i}=\prod_{j=1}^{n} \beta_{j}^{q a_{j i}-\delta_{j i}}
$$

If $\gamma_{i}=\prod_{k=1}^{n} \alpha_{k}^{c_{k i}^{\prime}}$ and $\delta_{j}=\prod_{k} \beta_{k}^{b_{k j}}$ these equations are equivalent to

$$
\gamma_{i}=\delta_{i}^{m_{i}}
$$

This equation can be solved for $\delta_{1}, \cdots, \delta_{n}$. Setting

$$
\beta_{j}=\prod_{k} \delta_{k}^{b_{k j}^{\prime}}
$$

we solve the original equations.

There is an exact sequence

$$
0 \rightarrow U_{K} \rightarrow W_{K / F} \stackrel{\mu}{\rightarrow} \mathbb{Z} \rightarrow 0
$$

such that $\mu(w)=1$ implies that $\sigma(w)$ is the Frobenius substitution in $\mathfrak{G}(K / F)$. Then $\mu(w)$ is 1 if and only if the transfer of $w$ in $C_{K}$ generates the prime ideal of $O_{K}$. The preceding discussion shows that under the isomorphism

$$
H_{1}\left(W_{K / F}, \widehat{L}\right) \leftrightarrow \operatorname{Hom}_{\mathfrak{G}(K / F)}\left(L, C_{K}\right)
$$

the image of $H_{1}\left(U_{K}, \widehat{L}\right)$ in $H_{1}\left(W_{K / F}, \widehat{L}\right)$ corresponds to $\operatorname{Hom}_{\mathfrak{G}(K / F)}\left(L, U_{K}\right)$. Thus the generalized character associated to an element of $H_{c}^{1}\left(W_{K / F}, \widehat{T}\right)$ is trivial on $\operatorname{Hom}_{\mathfrak{G}(K / F)}\left(L, U_{K}\right)$ if and only if that element is in the image of the inflation

$$
H^{1}(\mathbb{Z}, \widehat{L}) \rightarrow H^{1}\left(W_{K / F}, \widehat{L}\right) .
$$


The action of $\mathbb{Z}$ on $\widehat{L}$ is determined by that of $W_{K / F}$.

The sequence

$$
0 \rightarrow U_{K} \rightarrow C_{K} \stackrel{\nu}{\rightarrow} \mathbb{Z} \rightarrow 0
$$

in which $\nu(a)=1$ if and only if $a$ generates the prime ideal of $O_{K}$ splits as a sequence of $\mathfrak{G}(K / F)$-modules and leads to the exact sequence

$$
0 \rightarrow \operatorname{Hom}_{\mathfrak{G}(K / F)}\left(L, U_{K}\right) \rightarrow \operatorname{Hom}_{\mathfrak{G}(K / F)}\left(L, C_{K}\right) \rightarrow \operatorname{Hom}_{\mathfrak{G}(K / F)}(L, \mathbb{Z}) \rightarrow 0,
$$

and the generalized characters of $\operatorname{Hom}_{\mathfrak{G}(K / F)}\left(L, C_{K}\right)$ that are trivial on $\operatorname{Hom}_{\mathfrak{G}(K / F)}\left(L, U_{K}\right)$ are just the generalized characters of $\operatorname{Hom}_{\mathfrak{G}(K / F)}(L, \mathbb{Z})$, which is contained in $\operatorname{Hom}(L, \mathbb{Z})=\widehat{L}$.

If $\widehat{\lambda}$ is an invariant element of $\widehat{L}$ and $w$ belongs to $W_{K / F}$, consider the 1-cycle $x$, where $x(w)=\widehat{\lambda}$ while $x(u)=0$ if $u \neq w$. The image of the class of $x$ in $\operatorname{Hom}\left(L, C_{K}\right)$ is the homomorphism

$$
\lambda \rightarrow\left\{\prod_{\tau} \delta\left(w_{\tau}, w\right)\right\}^{\langle\lambda, \widehat{\lambda}\rangle} .
$$

Now $\prod_{\tau} \delta\left(w_{\tau}, w\right)$ is just the transfer of $w$. Applying $\nu$ we obtain the homomorphism $\mu(w) \hat{\lambda}$. Thus if $f$ is a 1 -cocycle on $W_{K / F}$ with values in $\widehat{T}$ associated to a 1 -cocycle on $\mathbb{Z}, \mu(w)=1, \widehat{\lambda}$ is an invariant element of $\widehat{L}$, and $\chi$ is the generalized character of $\widehat{L}^{\mathfrak{G}(K / F)}$ associated to $f$, then

$$
f(w)(\widehat{\lambda})=\chi(\lambda)
$$

Given $\chi$ we can take any extension of $\chi$ to $\widehat{L}$, take $f(w)$ to be that extension when $\mu(w)=1$, and extend $f$ to all of $W_{K / F}$ by the cocycle condition.

Suppose $\rho$ is a rational representation of the complex algebraic group $\widehat{T} \times \mathfrak{G}(K / F)$. Given $\chi$ we define a local $L$-function $L(s, \chi, \rho)$ by extending $\chi$ to $\widehat{L}$ and thus defining $t$ in $\widehat{T}$, choosing the Frobenius substitution $\sigma$ in $\mathfrak{G}(K / F)$, and finally setting

$$
L(s, \chi, \rho)=\frac{1}{\operatorname{det}\left(I-\rho(t \times \sigma)\left|\prod\right|^{s}\right)}
$$

if $\prod$ is a generator of the prime ideal of $O_{K}$.

But $f$ determines a homomorphism $\varphi$ of $W_{K / F}$ into $\widehat{T} \times \mathfrak{G}(K / F)$ :

$$
\varphi(w)=f(w) \times \sigma(w) .
$$

Thus $\rho \circ \varphi$ is a representation of $W_{K / F}$. The associated local $L$-function is

$$
L(s, \rho \circ \varphi)=\frac{1}{\operatorname{det}\left(I-\rho \circ \varphi(w)\left|\prod\right|^{s}\right)}
$$


if $\mu(w)=1$. Since $f(w)$ may be taken equal to $t$, the two functions are equal.

\section{References}

[1] E. Artin and J. Tate, Class Field Theory, Harvard, 1961.

[2] S. Lang, Rapport sur la Cohomologie des Groupes, New York, 1966.

[3] J.P. Serre, Corps Locaux, Hermann, Paris, 1962.

Institute For AdVANCED STUdy

Princeton, NJ 08540

E-mail address: rpl@ias.edu 